\title{
Revisiting Cyclic Voltammetry and Electrochemical Impedance Spectroscopy Analysis for Capacitance Measurements
}

\author{
Oumaïma Gharbi*, Mai T.T. Tran, Bernard Tribollet, Mireille Turmine and Vincent Vivier \\ Laboratoire Interfaces et Systèmes Electrochimiques (LISE), CNRS, UMR 8235, Sorbonne Université, \\ Paris, France
}

\begin{abstract}
The electric properties of an electrode can be evaluated by the determination of the capacitance. However, the literature reports a large panel of experimental protocols for capacitance determination, which involve either cyclic voltammetry (CV) or electrochemical impedance spectroscopy (EIS). Indeed, substantial discrepancies in the assumptions are made between both techniques as in $\mathrm{CV}$, the system is usually considered as ideal (and the current-scan rate relationship is assumed to remain linear at all frequencies), whereas in EIS, the system is usually considered as non-ideal and a CPE element is introduced. In this work, the electric properties of two electrode configurations, namely an Au blocking electrode and an Al electrode with a thin oxide film were investigated using EIS and CV. The capacitive domain was determined for both systems assuming the presence of an ideal and non-ideal system. The results collected from CV revealed a limited linearity between the current and scan rate for both electrode configurations. Finally, a CPE element was introduced to determine the capacitance from CV data and compared to EIS results.
\end{abstract}

Keywords: Constant phase element, Frequency dispersion, Supercapacitors, Capacitance, Double layer

*Corresponding author: oumaima.gharbi@upmc.fr 


\section{Introduction}

Cyclic voltammetry (CV) is a routine electrochemical technique used for the determination of electric properties of super capacitors or energy storage devices [1-3]. Typically, the capacitive charge is determined through the variation of the scan rate in a relatively constant (non-faradaic) current domain and is often used to estimate the performances of these electrodes. In fact, for ideal capacitors, the capacitive current is expected to follow a linear relationship with the scan rate, giving a characteristic rectangle voltammogram shape. Conversely, electrochemical impedance spectroscopy (EIS) is an electrochemical technique used for the determination of interfacial processes and also provides precise information on the double layer capacitance, and the oxide capacitance in the case of more complex systems[4-8]. For instance, when a metal-oxide/electrolyte interface is considered, EIS can provide information on the capacitive behaviour of the system in the case of an ideal capacitor. Indeed, at high frequencies, the contribution of the oxide layer capacity in series with the double layer capacitance can be determined whereas, in low frequencies, the contribution of the electric double layer can be extracted. Interestingly, the EIS measurement usually points out a non-ideal behaviour of the electrode due to frequency dispersion or time-constant dispersion and the used of constant phase element (CPE) to account for the capacitive behaviour of the interface [9]. At this point, it should be mentioned that the physical of the $\mathrm{CPE}$ has been discussed for decades in the literature [10-12] pointing out that the CPE is a mathematical alternative for simply describing the interface, but the physical justification is usually not realistic. This has led to the development of works in order to convert the CPE parameters into more realistic (from a physical point of view) capacitance $[13,14]$. In the following, the non-ideal terminology is used for an electrode that requires a CPE to describe the electrified interface as opposed to the use of a pure capacitance for an ideal behaviour.

Although both techniques are used to provide similar information on the capacitive characteristics of electrochemical interfaces, a clear correlation between the results obtained with each technique has not yet been clearly established. In particular, when a non-ideal behaviour for described by a CPE is invoked, the relationship between the capacitances obtained from the two techniques is not straightforward[15]. From a practical point of view, when a metal-oxide-electrolyte interface is considered, systems are not considered as ideal capacitors whereby, the oxide and double layer capacitances can be each described by a CPE element. However, cyclic voltammetry is known to be the typical measurement method for the estimation of capacitance and most of the work reported in the literature considers the behaviour of the electrochemical interface to be ideal, thus using a pure capacity to describe it.

In fact, recent work from Allagui et al. [16] revealed that the time-voltage relationship cannot be used when the system is considered as ideal. In their work, Allagui et al. introduced an alternative model (i.e. a fractional order equation) including a CPE element when cyclic voltammetry measurement was 
conducted on capacitors, and successfully validated the new expression with experimental results, corroborating the notion of non-ideal systems. Such research in fact raised a crucial point on the dependence of the measured capacitance in $\mathrm{CV}$ on the frequency range used for the measurement and calls into question on the accuracy of $\mathrm{CV}$ interpretation for capacitance determination.

Additionally, both techniques, CV and EIS are used for exploring the capacitance response of an electrode. However, the former allows accessing to the integral capacitance (measured as the integral of the current over the half cycle potential window) $[16,17]$ whereas the latter results in the measurement of the differential capacitance corresponding to the slope of the time voltage curve. Such differences in measurements should arise significant attention as both of the techniques are largely used to assess capacitor and super-capacitors properties.

In this work, electrochemical impedance spectroscopy and cyclic voltammetry were performed on wellknown systems showing capacitive behaviours on a wide frequency domain and over a wide potential range: a blocking electrode $(\mathrm{Au})$ and an electrode with a thin oxide film ( $\mathrm{Al})$. The capacitive charge was determined using cyclic voltammetry from the analysis of the conventional current to scan rate linear relationship and EIS measurement. Although, this data interpretation is valid in the broad context of an electrified interface, the results are discussed and compared to the relationship introduced by Allagui et al [16].

\section{Experimental}

In order to depict the impact of the electrochemical measurement (i.e. EIS or CV) and selected parameters such as scan rate or applied potential on the results, the electrochemical response of two different electrochemical systems involving two different electrolytes $\left(\mathrm{Na}_{2} \mathrm{SO}_{4}\right.$ and $\left.\mathrm{NaClO}_{4}\right)$ at $0.01 \mathrm{M}$ have been investigated herein. The response of a blocking electrode was studied using a $5 \mathrm{~mm}$ in diameter Au polycrystalline disk-electrode and the response of an $8 \mathrm{~mm}$ diameter Al disk-electrode was used to reproduce the electrode with a thin oxide film configuration. The counter electrode was a $\mathrm{Pt}$ gauze of about $16 \mathrm{~cm}^{2}$ which was placed in the bottom of the cell facing the rotating-disk electrode. No Luggin capillary was used to avoid changing current and potential distributions.

The thin oxide film formed on the aluminum electrode consisted was obtained by immersion of the electrode in the electrolyte for 30' (resulting in the formation of a passive film of a few nanometer - see below).

The experiments were conducted in Ar purged sodium sulphate $\left(\mathrm{Na}_{2} \mathrm{SO}_{4}\right)$ and sodium perchlorate $\left(\mathrm{NaClO}_{4}\right)$ solutions at $0.01 \mathrm{M}$. The solution was deaerated for $30^{\prime}$ ' before and during the experiment. Experiments with $\mathrm{NaClO}_{4}$ were only performed with the Au electrode to discard the effect of adsorbed species on the electrode. All experiments were realised using a Gamry Ref600+ potentiostat, a rotating 
disk electrode at $800 \mathrm{rpm}$ using a saturated mercury sulphate (MSE $-E^{0}=0.64 \mathrm{~V} / \mathrm{NHE}$ at $25^{\circ} \mathrm{C}$ ) reference electrode as well as a platinum grid as a counter electrode. The electrochemical cell was thermostatically controlled at $25.0 \pm 0.1{ }^{\circ} \mathrm{C}$ using a water recirculating bath.

Cyclic voltammetry measurement - Prior to each experiment, the electrode was cycled over the oxidation and reduction limits of the solvent. The capacitive domain of each system, which corresponds to the potential window whereby current variations are negligible was determined by a preliminary study with $\mathrm{CV}$ experiments. Once the potential window was determined, the CV were collected at increasing sweep rates varying between $1 \mathrm{mV} \mathrm{s}^{-1}$ to $2 \mathrm{~V} \mathrm{~s}^{-1}$. Conventionally, the capacitance of the system $(C)$ can be extracted from the following relationship

$$
i_{c}(t)=C \frac{d V}{d t}
$$

where $\mathrm{C}$ is the capacitance in $\mathrm{F} \mathrm{cm}{ }^{-2}, \mathrm{i}_{\mathrm{c}}$ is the capacitive current in $\mathrm{A} \mathrm{cm}{ }^{-2}$ and $\mathrm{dV} / \mathrm{dt}$ is the scan rate in $\mathrm{V} \mathrm{s}^{-1}$, or with the integral relationship

$$
C \frac{d V}{d t}=\frac{1}{\Delta V} \int i_{c}(t) d V
$$

Electrochemical impedance spectroscopy (EIS) measurement - Following CV experiments, EIS measurements were systematically collected at different potentials on the capacitive domain of the system (determined previously from the CV curves) every $50 \mathrm{mV}$. The measurements consisted of applying a $10 \mathrm{mV}$ rms amplitude perturbation in a frequency domain ranging from $100 \mathrm{kHz}$ to $10 \mathrm{mHz}$. Fitting of the Nyquist and electrolyte resistance corrected Bode representation (the module and phase angle were recalculated by correcting the real part of the impedance Z' with the electrolyte resistance $R_{e}$ [15]) were systematically realised for all the investigated systems and associated calculations for the double layer and oxide capacitance contributions were performed and are explained in details in the following section.

\section{Results and Discussion}

\subsection{EIS and CV response of a blocking electrode (Au)}

For ideal systems, the Nyquist representation of a blocking electrode should correspond to a vertical line translating the response of a pure capacitive behaviour. In this specific case, the capacitance value which corresponds to the double layer capacitance $\left(C_{d l}\right)$, can be determined from the imaginary part at high frequencies using the following equation:

$$
\left|Z^{\prime \prime}(\omega)\right|=1 / j \omega C_{d l}
$$


Where $\mathrm{Z}$ is the impedance in $\Omega \mathrm{cm}^{2}, j$ is the imaginary number, $\mathrm{C}_{\mathrm{dl}}$ expressed in $\mathrm{F} \mathrm{cm}^{-2}$ and $\omega$ the angular frequency in $\mathrm{rad} \mathrm{s}^{-1}$.

However experimentally, the Nyquist plot of an Au electrode shows a non-ideal behaviour at the high frequency domain revealing a CPE behaviour as shown in Figure 1. This is better evidenced on the Bode representation, where the electrolyte resistance corrected phase angle is almost a constant at values lower than $90^{\circ}$ (Figure 1 (c)) in the frequency range between $4 \mathrm{kHz}$ and $10 \mathrm{~Hz}$, thus allowing a graphical evaluation of the CPE parameter $(\alpha=0.9)$ from the constant value of the corrected phase angle in the high frequency domain. In this case, the impedance $\mathrm{Z}$ is defined by the following relationship:

$$
Z(\omega)=1 /(j \omega)^{\alpha} Q
$$

with $\alpha$ and $\mathrm{Q}$ as CPE parameters. It should be noted that the very high frequency domain shows a frequency dispersion due to the geometry of the electrode as demonstrated in different works [18-21]. In fact, when a CPE element is used to fit the experimental data, the results reveal $\alpha$ varying between 0.7 and 1. In the case of a double layer capacitance, the capacitance value can be determined using Brug's formula [13], which accounts for the surface distribution of the impedance[14] and expresses as

$$
C_{d l}=Q^{1 / \alpha}\left(1 / R_{e}+1 / R_{c t}\right)^{(\alpha-1) / \alpha}
$$

where $R_{c t}$ is the charge transfer resistance, expressed in $\Omega \mathrm{cm}^{2}$ (in this system, $1 / R_{c t}$ value was considered as negligible as $R_{c t}$ value was considered as infinite). For comparison purposes, the capacitance was calculated following both assumptions (i.e. assuming an ideal capacitive behaviour with Eq. 3 and using a CPE element to consider the dispersion at high frequencies with Eqs. 4 and 5) for each EIS scan and reported as a function of the applied potential as shown in Figure 2. The results clearly reveal a significant variation (about $30 \%$ ) of the capacitance as a function of the frequency when the system is considered as an ideal capacitor, as previously reported by several authors [22-24]. The difference in $C_{d l}$ value at two different frequencies when the system is considered as an ideal capacitor clearly emphasise the error made with this assumption. Conversely, the $C_{d l}$ value does not vary significantly over the capacitive potential window investigated in this work. As an example to illustrate the analogy between scan rate utilised in $\mathrm{CV}$ and frequencies in EIS, a measurement conducted at a frequency $f=$ $10 \mathrm{~Hz}$ with an amplitude of $\Delta \mathrm{E}=10 \mathrm{mV}$ would correspond to a $100 \mathrm{mV} \mathrm{s}^{-1}$ scan rate.

To further validate our assumption, cyclic voltammogram of an ideal and non-ideal capacitors were simulated and compared to our experimental data (i.e. $\mathrm{CV}$ of the $\mathrm{Au}$ electrode) and the results are illustrated in Figure 3. The simulated scans were produced using the linear current - scan rate relationship according to equation (1), for describing the capacitive-like behaviour of the interface, as the non-ideal capacitors scans were simulated using the equations introduced by Allagui et al. [16] 


$$
i_{c}(t)=Q \frac{d^{\alpha} V(t)}{d t^{\alpha}}
$$

It should be noted that for $\alpha=1$, equation (6) expresses the usual condition for an ideal capacitance. Therefore, the effective capacitance $C_{\text {eff }}$ can be determined using the following equation [16]:

$$
C_{e f f}=\frac{Q}{\Gamma(3-\alpha)} t^{1-\alpha}
$$

where, $\Gamma$ is the usual mathematical gamma function, Q and $\alpha$ the CPE parameters and $t$ the time in $\mathrm{s}$. Concomitantly, cyclic voltammetry experiments were conducted on the Au electrode in $0.01 \mathrm{M} \mathrm{Na}_{2} \mathrm{SO}_{4}$ at over 3 orders of magnitude for the scan rate $\left(1 \mathrm{mV} \mathrm{s}^{-1}\right.$ to $\left.2 \mathrm{~V} \mathrm{~s}^{-1}\right)$. The simulation results, obtained for $5 \mathrm{mV} \mathrm{s}^{-1}, 500 \mathrm{mV} \mathrm{s}^{-1}$ and $50 \mathrm{~V} \mathrm{~s}^{-1}$, reveal a significant difference in the curve shape when the system is considered as ideal or non-ideal. In fact, when the system is considered as an ideal capacitor and equation (1) is used, the CV curves exhibit perfect square shapes represented by the black line, whereas when equation (6) is considered, the shape of the CV curves (red line) closely resemble the experimental results obtained on the Au electrode. In addition, the difference in the current density between the "ideal and non-ideal" capacitor curves seems to decrease with increasing scan rate, indicating a scan rate dependence in the analysis when the system is considered as ideal, in agreement with the frequencydependent capacitance observed when a pure capacitance is used in place of a CPE [22]. This is a crucial point which deserves to be considered and discussed as the results clearly depend on the scan rate (which is similar to EIS analysis, where different capacitance values are determined at different frequencies). Such difference in variation is corroborated in Figure 4 where are represented the variation of the capacitive current as a function of the scan rate determined experimentally (Figure 4 (a)) and simulated using equation 1 and 4 (Figure 4 (b)) and the capacitance as a function of $\alpha$ (Figure 4 (c)). When the system is considered as ideal, the capacitive current density varies linearly (following equation 1), whereas once a CPE is considered, a slight variation in the linearity is observed between low and high scan rate range as illustrated in Figure 4(a). In fact, such variation in linearity is also readily observed in the experimental results, with an increase of the slope value (by a factor of $\sim 2$ ) at increasing scan rates. In addition, the capacitance varies considerably with $\alpha$ as illustrated in Figure 4 (c) which represents the variation of $C_{\text {eff }}$ as a function of time as described by equation 7 for two selected $\alpha$ values $\left(\alpha=0.9\right.$ and 0.85 ). In fact, for two different $\alpha$ values considered, in low scan rate range, $C_{\text {eff } \alpha=0.85}$ is lower than $C_{\text {eff } \alpha=0.9}$, however, as the scan rate increases, $C_{\text {eff } \alpha=0.85}$ increases with a larger slope, yielding to an inversion at $t=2 \mathrm{~s}$ which would correspond to a scan rate of $500 \mathrm{mV} \mathrm{s}^{-1}$ for $1 \mathrm{~V}$ domain amplitude. In fact, $C_{\text {eff } a=0.85}$ values become higher at high scan rate ranges, which demonstrate the time dependence of $C_{\text {eff }}$ measurement. These results show that the CPE parameters can either be obtained from the analysis of the EIS spectrum in a defined frequency domain or, similarly, using cyclic voltammetry at different scan rates. Interestingly, Eq. 7 devised by Allagui et al. [16] then allowed a capacitance to de determined. 
In order to discard the impact of adsorbed species on the electrodes, the CV and EIS response of an $\mathrm{Au}$ electrode was determined in a $0.01 \mathrm{M} \mathrm{NaClO}_{4}$ solution $\left(\mathrm{ClO}_{4}{ }^{-}\right.$anions are known to less adsorb on the surface in regards to $\mathrm{SO}_{4}{ }^{2-}$ ) as illustrated in Figures 5 (a) and (b). The CV were collected and EIS results are similar to the experiment conducted in $\mathrm{Na}_{2} \mathrm{SO}_{4}$ whereby the $\mathrm{CV}$ shapes are very similar to the simulation performed using a CPE element Figure 5 (a). In addition, the Nyquist plot demonstrates the non-ideal response at the high frequency domain corroborating the CPE behaviour as described for the response of the electrode in $0.01 \mathrm{M} \mathrm{Na}_{2} \mathrm{SO}_{4}$ shown in Figure 1.

\subsection{EIS and CV response of an electrode with a thin oxide film (Al)}

In this work, it was of interest to investigate a system involving a thin oxide film and determine the contribution of the oxide capacitance $\left(C_{o x}\right)$ on the capacitive response deduced from the $\mathrm{CV}$ measurement. In this section, particular attention is given to the electrochemical response of an electrode with a thin oxide film, and an attempt to correlate EIS measurement to CV analysis is presented. Following the same approach, EIS scans were performed in $0.01 \mathrm{M} \mathrm{Na}_{2} \mathrm{SO}_{4}$ at potentials varying between $-2 \mathrm{~V}_{\mathrm{MSE}}$ to $-1.3 \mathrm{~V}_{\mathrm{MSE}}$ and the Nyquist and electrolyte resistance corrected Bode representation are given in Figure 6. It is important to note that the cathodic branch in this potential window was considered negligible and allowed to use the equivalent circuit presented in the following section. The shape of the impedance diagram is characteristic of a capacitive response from the presence of a thin oxide film on the Al surface. In fact, the CPE behaviour is clearly distinguishable on the modified Bode representation with a constant phase angle close to $80^{\circ}$ between $100 \mathrm{~Hz}$ and $2 \mathrm{~Hz}$. At high frequency however, the phase angle decreases and increases due to geometry-induced current and potential distributions of the electrode, which is ascribed to the Ohmic contribution of the impedance as described in [21]. In the case of oxide films, such CPE response is attributed to a normal time constant distribution caused by variations in resistivity within the oxide film [14]. The electrical representation of the Al interface is then described as a decomposition in series of the impedance of the film in series with a CPE element ascribed to the double layer contribution as shown in Figure 7, which was used to realise the fitting of the impedance diagrams of $\mathrm{Al}$ obtained in $0.01 \mathrm{M} \mathrm{Na}_{2} \mathrm{SO}_{4}$. For this electrode configuration, the capacitance is described by the following relation:

$$
\frac{1}{C_{e f f}}=\frac{1}{C_{o x}}+\frac{1}{C_{d l}}
$$

In addition, the response of the film can be described by a power-law model, accounting for a resistivity profile along the thickness of the oxide film:

$$
Z_{f i l m}(\omega)=\int_{0}^{\delta_{o x}}\left(\rho(x) / 1+j \omega \varepsilon \varepsilon_{0} \rho(x)\right) d x
$$


where $\varepsilon$ is the oxide permittivity ( $\varepsilon=11.5$ for the aluminium oxide), $\varepsilon_{0}$ is the vacuum permittivity $\left(\varepsilon_{0}=\right.$ $\left.8.8542 \times 10^{-14} \mathrm{~F} \mathrm{~cm}^{-1}\right), \rho(x)$ is the resistivity distribution inside the oxide film (expressed with a power law[25,26]), and $\delta_{o x}$ is the film thickness (cm). Conversely, the double layer is described by a CPE as defined in equation (2) for which the following parameters were determined from the fitting $\alpha=0.87$ and $Q=18 \times 10^{-6} \mathrm{Fs}^{\alpha-1} \mathrm{~cm}^{-2}$. At high frequencies, $C_{o x}$ and $C_{d l}$ are both measured (with $1 / C_{o x}>>1 / C_{d l}$ ) whereas, in low frequencies, equation (9) tends to a resistance allowing the determination of $C_{d l}$. The oxide film capacitance can be determined from the extrapolation of the real capacitance at infinite frequency using the complex capacitance representation [8] as represented in Figure 8. Interestingly, the high frequency limit of the capacitance tends towards $C_{\text {lim }}$ (which correspond to the $C_{o x}$ ) value remains constant within this potential window with a value of $1.15 \mu \mathrm{F} \mathrm{cm}^{-2}$ can be determined on the real axis and an oxide thickness of $\sim 9 \mathrm{~nm}$, revealing that the $C_{o x}$ is independent of the potential within this range and is in good agreement with typical $C_{o x}$ values reported in the literature [7,8]and about 1 order of magnitude smaller than the usual value for a double layer capacitance. This result also justifies the assumption that in the high frequency domain, the double layer capacitance (in series with the capacitance of the oxide film) can be neglected.

In a similar manner, the CPE parameters corresponding to the double layer were collected from the fitting and the doubler layer capacitance was determined using Brug's relation (equation (5)) for each impedance scan collected from $-2 \mathrm{~V}_{\mathrm{MSE}}$ to $-1.3 \mathrm{~V}_{\mathrm{MSE}}$ (this potential range correspond to the capacitive domain of the system). The results, displayed in Figure 9, reveal that the calculated $C_{d l}$ value for each EIS scan does not vary significantly with the potential (i.e. starts at $26.2 \mu \mathrm{F} \mathrm{cm}$ and ends at $24.2 \mu \mathrm{F}$ $\mathrm{cm}^{-2}$ ), which is very similar to what was observed (in terms of trend) with the Au electrode. Conversely, when the capacitance is determined using CV measurement, as displayed in Figure 10, a clear increase in $C_{d l}$ value can be observed between high scan rate and low scan rate ranges. It varies from $15 \mu \mathrm{F} \mathrm{cm}{ }^{-2}$ for high scan rate, up to $20 \mu \mathrm{F} \mathrm{cm}{ }^{-2}$ for low scan rate (i.e. about $25 \%$ variation) in agreement with the time dependent expression reported in equation (7). However, discrepancies in $\mathrm{C}_{\mathrm{dl}}$ values between the values calculated from EIS and CV (around 20 to $40 \%$ difference) is observed. The origin of such discrepancies stems from equation 7 , which was not built on a physical description of the origin of the CPE but rather a mathematical solution of the equation which does not consider the physical and chemical properties of the system. Although such scan rate range only corresponds to low frequency values, the frequency dependence of the capacitance significantly alters the accuracy of the data, varying from a factor of 2 to 3 from low to high scan rate when extending the scan-rate range to a greater domain to the results presented in Figure 10. 
The notion of frequency dependence on the capacitance measurement of electrodes with thin oxide film has been previously reported in the literature. In fact, several researchers reported discrepancies in the calculated capacitance values, and revealed that it was due to the erroneous assumption that the system considered behaves like an ideal capacitor. For impedance measurement, the constant phase element has been introduced to describe the non-ideal response of electrodes. However, for identical electrodes, the capacitance measurement determination in cyclic voltammetry using the linear scan rate-current relationship assumes an ideal response of the system. In fact, as shown by Harrington and Devine [19] and illustrated in Figure 2, the capacitance value varies considerably with the frequency. Such frequency dependence is expected to be observed during cyclic voltammetry measurements, although rarely seen as $C_{\text {eff }}$ analysis usually occurs at scan rates between 1 and $100 \mathrm{mV} \mathrm{s}^{-1}$ [27-29] and has also been reported for vertical alignments of graphene sheets for higher scan rates [30]. In fact, in the case of systems involving an oxide and a double layer capacitance, it is critical to select appropriate frequency ranges for $\mathrm{CV}$ analysis as it will either enhance $C_{d l}$ response (low frequencies) or $C_{o x}$ response (high frequencies) if the dielectric properties are determined using cyclic voltammetry. As an example, the $\mathrm{CV}$ analysis conducted on the $\mathrm{Al}$ electrode clearly reveals a response from the $C_{d l}$ which is in good agreement with the scan rate selected in this study (which corresponds to a low frequency range).

In order to verify whether $C_{\text {eff }}$ expression presented by Allagui et al. would correlate with our experimental CV and EIS results, the capacitance for each scan rate was calculated using $C_{\text {eff }}$ expression (equation (7)) for the Al electrode. The $Q$ and $\alpha$ used for the calculations were determined from the EIS measurements and discussed previously $\left(\alpha=0.87\right.$ and $\left.Q=18 \times 10^{-6} \mathrm{Fs}^{\alpha-1} \mathrm{~cm}^{-2}\right)$ and the time was expressed as the scan rate divided by the $\mathrm{CV}$ domain amplitude (i.e. $700 \mathrm{mV}$ for these experiments). The results illustrated in Figure 11, show values in good correlation for scan rates between $100 \mathrm{mVs}^{-1}$ and $2 \mathrm{Vs}^{-1}$ to what was determined experimentally via $\mathrm{CV}$. The evolution of $C_{e f f}$ as a function of the scan rate correlates both techniques and affirms the limited linearity between the capacitive current density and scan rate, supporting the presence of a CPE element in the relationship. However, the impact of $\alpha$ (which may vary with the system) on $C_{\text {eff }}$ still needs further investigation. 


\section{Conclusion}

In this work, the capacitive response of two electrode configurations (namely a Au blocking electrode and an $\mathrm{Al}$ electrode with a thin oxide film) was studied using cyclic voltammetry measurement and electrochemical impedance spectroscopy. For both systems, it was demonstrated that during CV measurements, a non-linear relationship between the scan rate and the capacitive current occurs for large scan rate ranges (i.e. more than two order of magnitude of scan rate range) which reveals a non-ideal behaviour of the electrochemical interface.

In $\mathrm{CV}$ measurements, the calculated capacitance predominantly stems from the double layer capacitance for the considered scan rate ranges (low frequency range). Additionally, it was shown that the value of the determined capacitance is scan-rate dependent. Such experimental results highlight the arbitrary character of the scan rate ranges used in the literature as they usually remain in low frequency range. In fact, careful selection of scan rate is critical and should be adapted to the electrode considered, particularly for systems involving a thin oxide film as the $C_{d l}$ or the $C_{o x}$ response depends on the frequency range. Similarly, the EIS response shows that the capacitance determination cannot be performed without a large error when a non-ideal behaviour is experimentally obtained. From a physical point of view, such outcome in this frequency domain is the results of observations made in the selected time domain.

The mathematical development devised by Allagui et al. [16] further validates the presence of non-ideal capacitive behaviour for both systems and provides promising correlation between EIS and CV results. The calculated $C_{\text {eff }}$ values using their relationship and $\alpha$ values collected from EIS experiments reveal to be very similar at three different scan rate ranges. However, future work is required to explain the effect of $\alpha$ value on $C_{\text {eff }}$, which indeed let the door opened to the general question on the physical meaning of a CPE for a surface distribution as a double layer capacitance, particularly in the broader context of supercapacitors or energy storage properties measurements. 


\section{References}

[1] W.G. Pell, B.E. Conway, Analysis of power limitations at porous supercapacitor electrodes under cyclic voltammetry modulation and dc charge, in: J. Power Sources, 2001: pp. 57-67. doi:10.1016/S0378-7753(00)00682-0.

[2] V. Augustyn, P. Simon, B. Dunn, Pseudocapacitive oxide materials for high-rate electrochemical energy storage, Energy Environ. Sci. 7 (2014) 1597-1614. doi:10.1039/c3ee44164d.

[3] P. Simon, Y. Gogotsi, Materials for electrochemical capacitors, Nat. Mater. 7 (2010) 138-147. doi:10.1142/9789814317665_0021.

[4] B.E. Conway, Transition from "supercapacitor" to "battery" behavior in electrochemical energy storage, J. Electrochem. Soc. 138 (1991) 319-327. doi:10.1149/1.2085829.

[5] E. Frackowiak, F. Béguin, Carbon materials for the electrochemical storage of energy in capacitors, Carbon N. Y. 39 (2001) 937-950. doi:10.1016/j.matlet.2012.07.085.

[6] P.L. Taberna, P. Simon, J.F. Fauvarque, Electrochemical Characteristics and Impedance Spectroscopy Studies of Carbon-Carbon Supercapacitors, J. Electrochem. Soc. 150 (2003) A292. doi:10.1149/1.1543948.

[7] T.T.M. Tran, B. Tribollet, E.M.M. Sutter, New insights into the cathodic dissolution of aluminium using electrochemical methods, Electrochim. Acta. 216 (2016) 58-67. doi:10.1016/j.electacta.2016.09.011.

[8] M. Benoit, C. Bataillon, B. Gwinner, F. Miserque, M.E. Orazem, C.M. Sánchez-Sánchez, B. Tribollet, V. Vivier, Comparison of different methods for measuring the passive film thickness on metals, Electrochim. Acta. 201 (2016) 340-347. doi:10.1016/j.electacta.2015.12.173.

[9] G.J. Lee, S. Il Pyun, Effect of microcrystallite structures on electrochemical characteristics of mesoporous carbon electrodes for electric double-layer capacitors, Electrochim. Acta. 51 (2006) 3029-3038. doi:10.1016/j.electacta.2005.08.037.

[10] E. Barsoukov, J.R. Macdonald, Impedance spectroscopy theory, experiment, and applications, Second, Willey \& Sons, 2005. doi:10.5860/choice.43-0327.

[11] A. Sadkowski, Time domain responses of constant phase electrodes, Electrochim. Acta. 38 (1993) 2051-2054. doi:10.1016/0013-4686(93)80339-2.

[12] A. Sadkowski, On the ideal polarizability of electrodes displaying cpe-type capacitance dispersion, J. Electroanal. Chem. 481 (2000) 222-226. doi:10.1016/S0022-0728(99)00481-7.

[13] G.J. Brug, A.L.G. van den Eeden, M. Sluyters-Rehbach, J.H. Sluyters, The analysis of electrode impedances complicated by the presence of a constant phase element, J. Electroanal. Chem. 176 (1984) 275-295. doi:10.1016/S0022-0728(84)80324-1.

[14] B. Hirschorn, M.E. Orazem, B. Tribollet, V. Vivier, I. Frateur, M. Musiani, Determination of effective capacitance and film thickness from constant-phase-element parameters, Electrochim. Acta. 55 (2010) 6218-6227. doi:10.1016/j.electacta.2009.10.065.

[15] M.E. Orazem, B. Tribollet, Electrochemical Impedance Spectroscopy, 2008. doi:10.1002/9780470381588.

[16] A. Allagui, T.J. Freeborn, A.S. Elwakil, B.J. Maundy, Reevaluation of Performance of Electric Double-layer Capacitors from Constant-current Charge/Discharge and Cyclic Voltammetry, Sci. Rep. 6 (2016). doi:10.1038/srep38568.

[17] T. Tooming, T. Thomberg, H. Kurig, A. Jänes, E. Lust, High power density supercapacitors based on the carbon dioxide activated d-glucose derived carbon electrodes and 1-ethyl-3methylimidazolium tetrafluoroborate ionic liquid, J. Power Sources. 280 (2015) 667-677. doi:10.1016/j.jpowsour.2015.01.157.

[18] J. Newman, Frequency Dispersion in Capacity Measurements at a Disk Electrode, J. Electrochem. Soc. 117 (1970) 198. doi:10.1149/1.2407464.

[19] V.M.-W. Huang, V. Vivier, M.E. Orazem, N. Pébère, B. Tribollet, The Apparent ConstantPhase-Element Behavior of an Ideally Polarized Blocking Electrode, J. Electrochem. Soc. 154 (2007) C81. doi:10.1149/1.2398882.

[20] V.M.-W. Huang, V. Vivier, I. Frateur, M.E. Orazem, B. Tribollet, The Global and Local Impedance Response of a Blocking Disk Electrode with Local Constant-Phase-Element 
Behavior, J. Electrochem. Soc. 154 (2007) C89. doi:10.1149/1.2398889.

[21] O. Gharbi, A. Dizon, M.E. Orazem, M.T.T. Tran, B. Tribollet, V. Vivier, From frequency dispersion to ohmic impedance: A new insight on the high-frequency impedance analysis of electrochemical systems, Electrochim. Acta. 320 (2019). doi:10.1016/j.electacta.2019.134609.

[22] S.P. Harrington, T.M. Devine, Analysis of Electrodes Displaying Frequency Dispersion in MottSchottky Tests, J. Electrochem. Soc. 155 (2008) C381. doi:10.1149/1.2929819.

[23] S.P. Harrington, F. Wang, T.M. Devine, The structure and electronic properties of passive and prepassive films of iron in borate buffer, Electrochim. Acta. 55 (2010) 4092-4102. doi:10.1016/j.electacta.2009.11.012.

[24] A.G. Breuhaus-Alvarez, J.L. Dimeglio, J.J. Cooper, C.R. Lhermitte, B.M. Bartlett, Kinetics and Faradaic Efficiency of Oxygen Evolution on Reduced H x WO 3 Photoelectrodes, J. Phys. Chem. C. 123 (2019) 1142-1150. doi:10.1021/acs.jpcc.8b11777.

[25] B. Hirschorn, M.E. Orazem, B. Tribollet, V. Vivier, I. Frateur, M. Musiani, Constant-PhaseElement Behavior Caused by Resistivity Distributions in Films, J. Electrochem. Soc. 157 (2010) C458. doi:10.1149/1.3499565.

[26] B. Hirschorn, M.E. Orazem, B. Tribollet, V. Vivier, I. Frateur, M. Musiani, Constant-PhaseElement Behavior Caused by Resistivity Distributions in Films, J. Electrochem. Soc. 157 (2010) C452. doi:10.1149/1.3499564.

[27] Y. Li, L. Cao, L. Qiao, M. Zhou, Y. Yang, P. Xiao, Y. Zhang, Ni-Co sulfide nanowires on nickel foam with ultrahigh capacitance for asymmetric supercapacitors, J. Mater. Chem. A. 2 (2014) 6540-6548. doi:10.1039/c3ta15373h.

[28] A. Yu, I. Roes, A. Davies, Z. Chen, Ultrathin, transparent, and flexible graphene films for supercapacitor application, Appl. Phys. Lett. 96 (2010) 198-201. doi:10.1063/1.3455879.

[29] H.B. Li, M.H. Yu, F.X. Wang, P. Liu, Y. Liang, J. Xiao, C.X. Wang, Y.X. Tong, G.W. Yang, Amorphous nickel hydroxide nanospheres with ultrahigh capacitance and energy density as electrochemical pseudocapacitor materials, Nat. Commun. 4 (2013) 1-7. doi:10.1038/ncomms2932.

[30] Y. Yoon, K. Lee, S. Kwon, S. Seo, H. Yoo, S. Kim, Y. Shin, Y. Park, D. Kim, J.Y. Choi, H. Lee, Vertical alignments of graphene sheets spatially and densely piled for fast ion diffusion in compact supercapacitors, ACS Nano. 8 (2014) 4580-4590. doi:10.1021/nn500150j. 
(a)
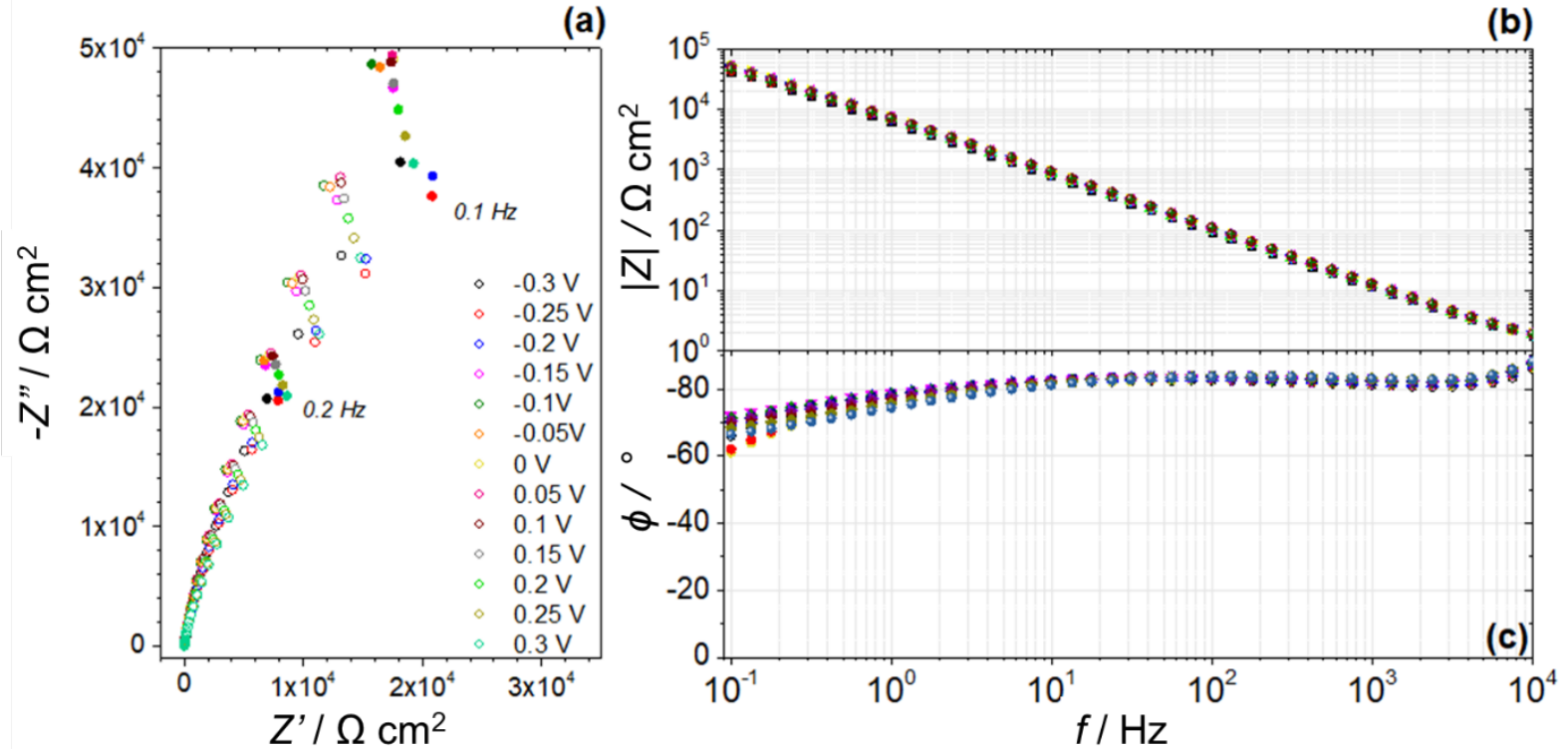

Figure 1: Nyquist (a), electrolyte resistance corrected Bode representation ((b) and (c)) of a blocking ( $\mathrm{Au}$ ) electrode in $0.01 \mathrm{M} \mathrm{Na}_{2} \mathrm{SO}_{4}$ collected at potential varying between -0.3 to $0.3 \mathrm{~V}_{\text {MSE }}$. 


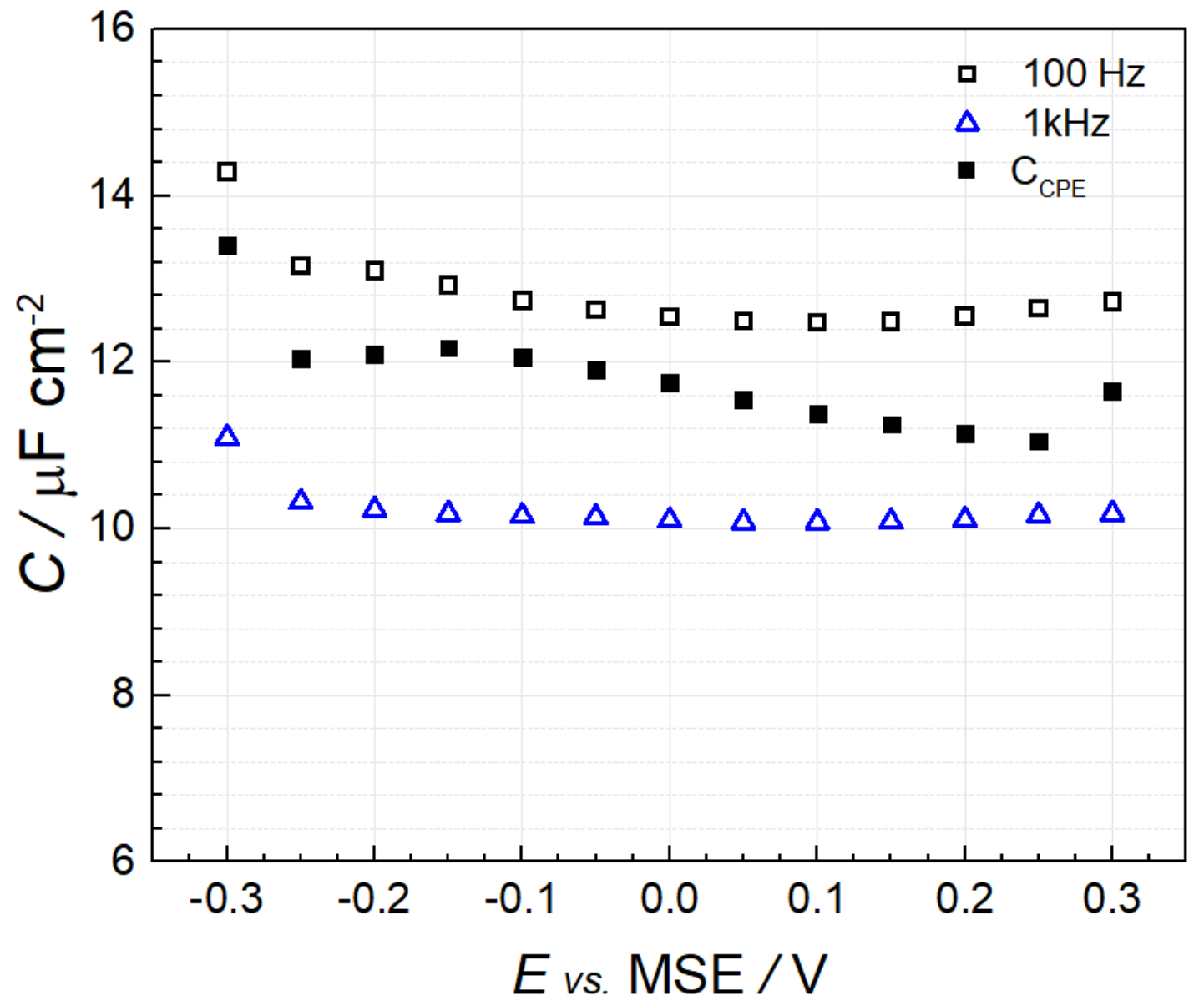

Figure 2: Evolution of the double layer capacitance of an Au electrode as a function of the potential, calculated assuming an ideal $\left(C_{100 \mathrm{~Hz}}\right.$ and $\left.C_{1 k \mathrm{~Hz}}\right)$ and non-ideal system $\left(C_{C P E}\right)$. The capacitance at two frequencies $(100 \mathrm{~Hz}$ and $1 \mathrm{kHz})$ were determined from the imaginary part of the impedance $\mathrm{Z}$ ' using Eq. 3. When the system was considered as non-ideal, a CPE element was used to describe the system Z' (using Eq. 4) and Brug's formula (Eq. 5) was used to calculate $C_{C P E}$. The results highlight the frequency dependence of $C$ when an ideal system is considered. 
(a)
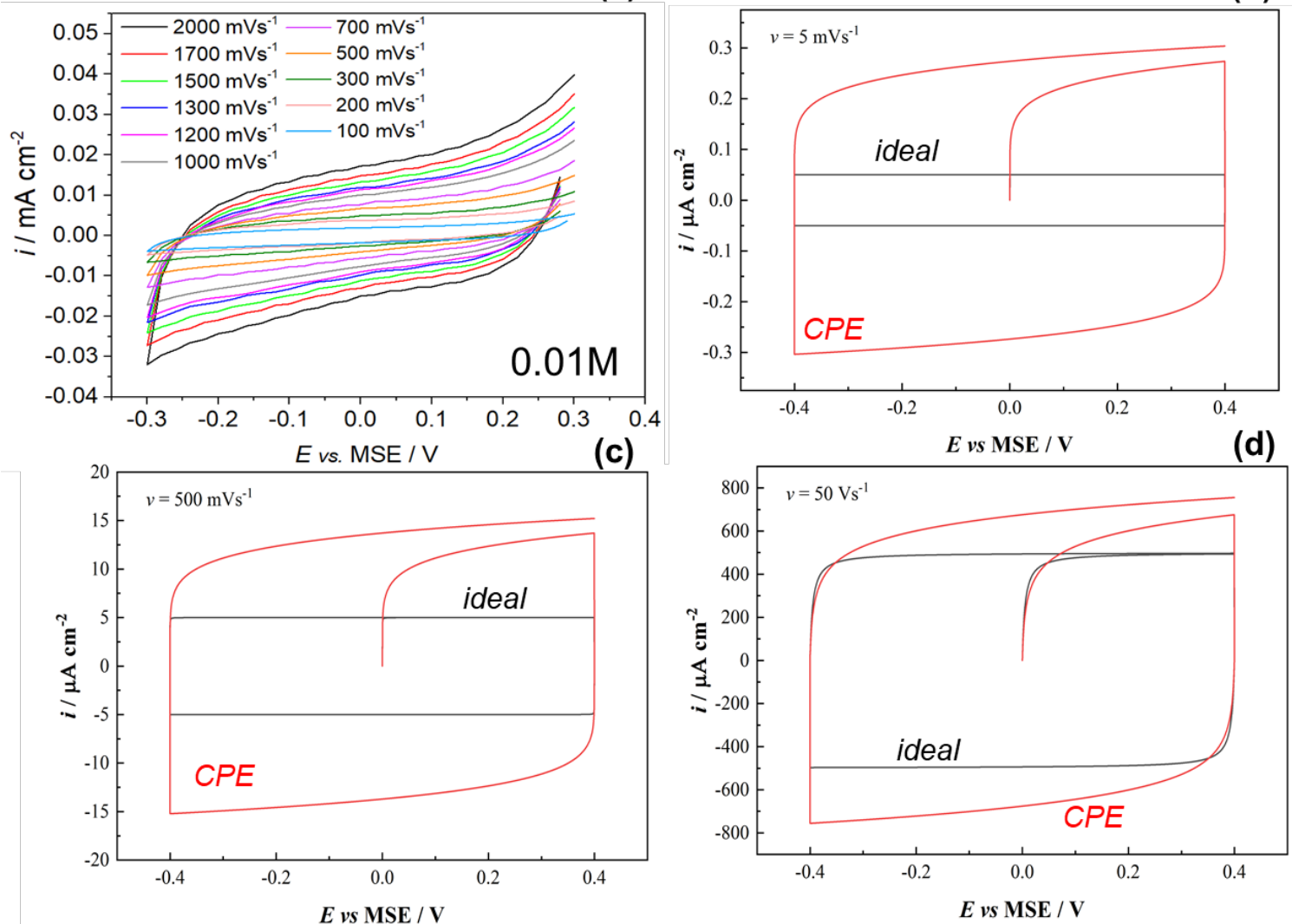

Figure 3: Comparison between experimental (a) cyclic voltammogram curves obtained in $0.01 \mathrm{M}$ $\mathrm{Na}_{2} \mathrm{SO}_{4}$ of an Au electrode and simulated CV (b), (c) and (d) assuming an ideal and non-ideal (CPE) capacitor using equations 1 and 6, with the following parameters $Q=3010^{-6} \mathrm{Fs}^{\alpha-1} \mathrm{~cm}^{-2} ; \alpha=0.9$ for the $\mathrm{CPE}$ and $C=10^{-5} \mathrm{~F} \mathrm{~cm}^{-2}$ for the ideal capacitance. The results indicate a similarity in the $\mathrm{CV}$ shape between the experimental and the simulated CV when a CPE element is introduced, reinforcing the notion that the system behaves as a non-ideal capacitor. 
(a)

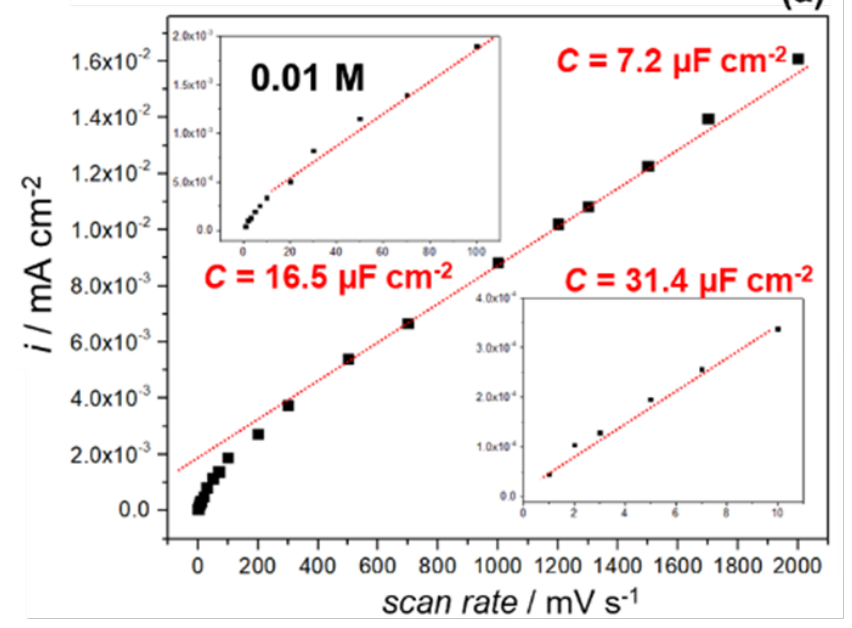

(b)

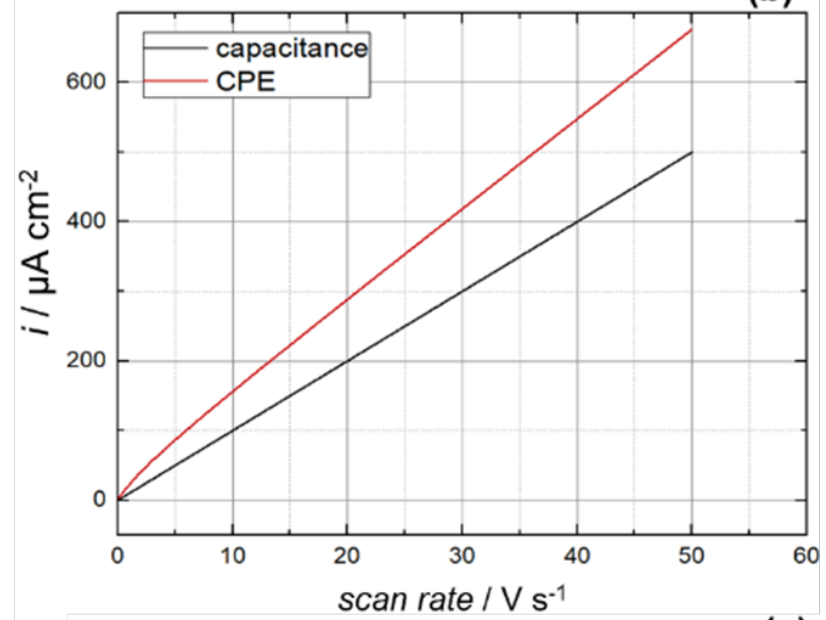

(c)

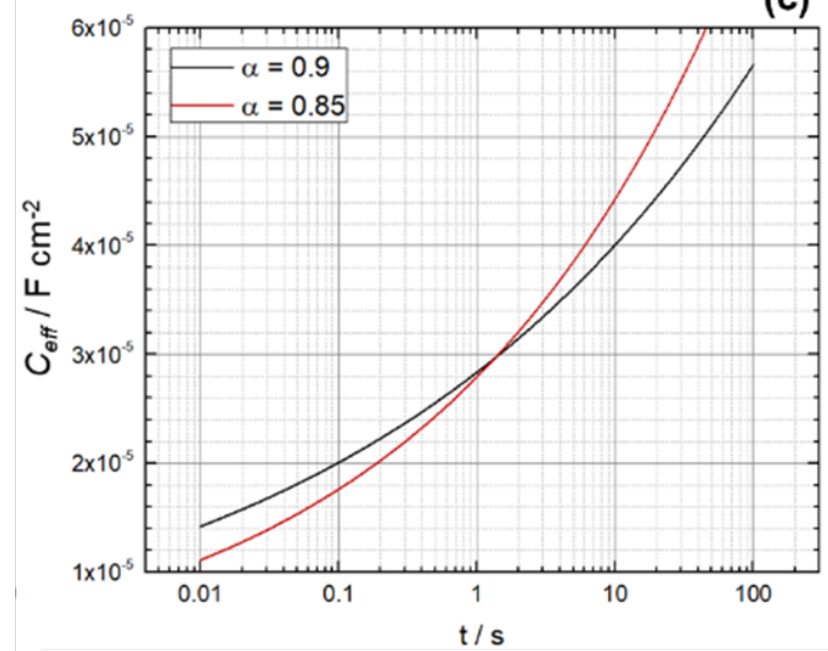

Figure 4: Graphic representation of the capacitive current density of an Au electrode in $0.01 \mathrm{M} \mathrm{Na}_{2} \mathrm{SO}_{4}$ solution as a function of the scan rate (a), the evolution of the capacitive current density simulated using equation (1) and (4) as a function of the scan rate (b) and the variation of the effective capacitance as a function of time for two different $\alpha$ factors $\left(Q=3010^{-6} \mathrm{Fs}^{\alpha-1} \mathrm{~cm}^{-2} ; \alpha=0.9\right.$ and 0.85$)$ (c). The results clearly show a difference in linearity as the scan rate increases yielding to an increase of the slope $\left(C_{d l}\right)$ by a factor of $\sim 2$. 
(a)
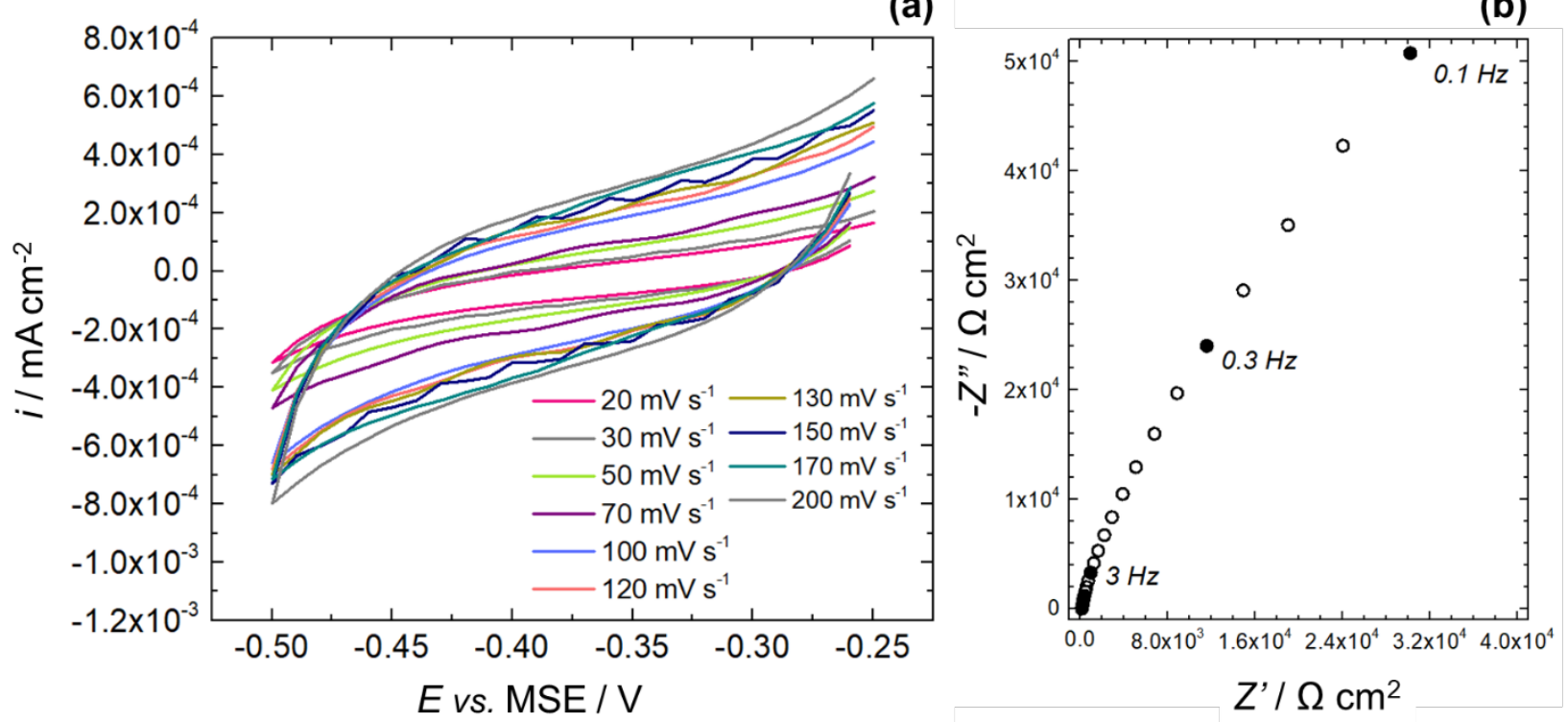

Figure 5: Experimental CV curves collected on a blocking electrode $(\mathrm{Au})$ in $0.01 \mathrm{M} \mathrm{NaClO}_{4}$ (a) and the Nyquist representation collected at $E=-0.3 \mathrm{~V}_{\mathrm{MSE}}(\mathrm{b})$. 

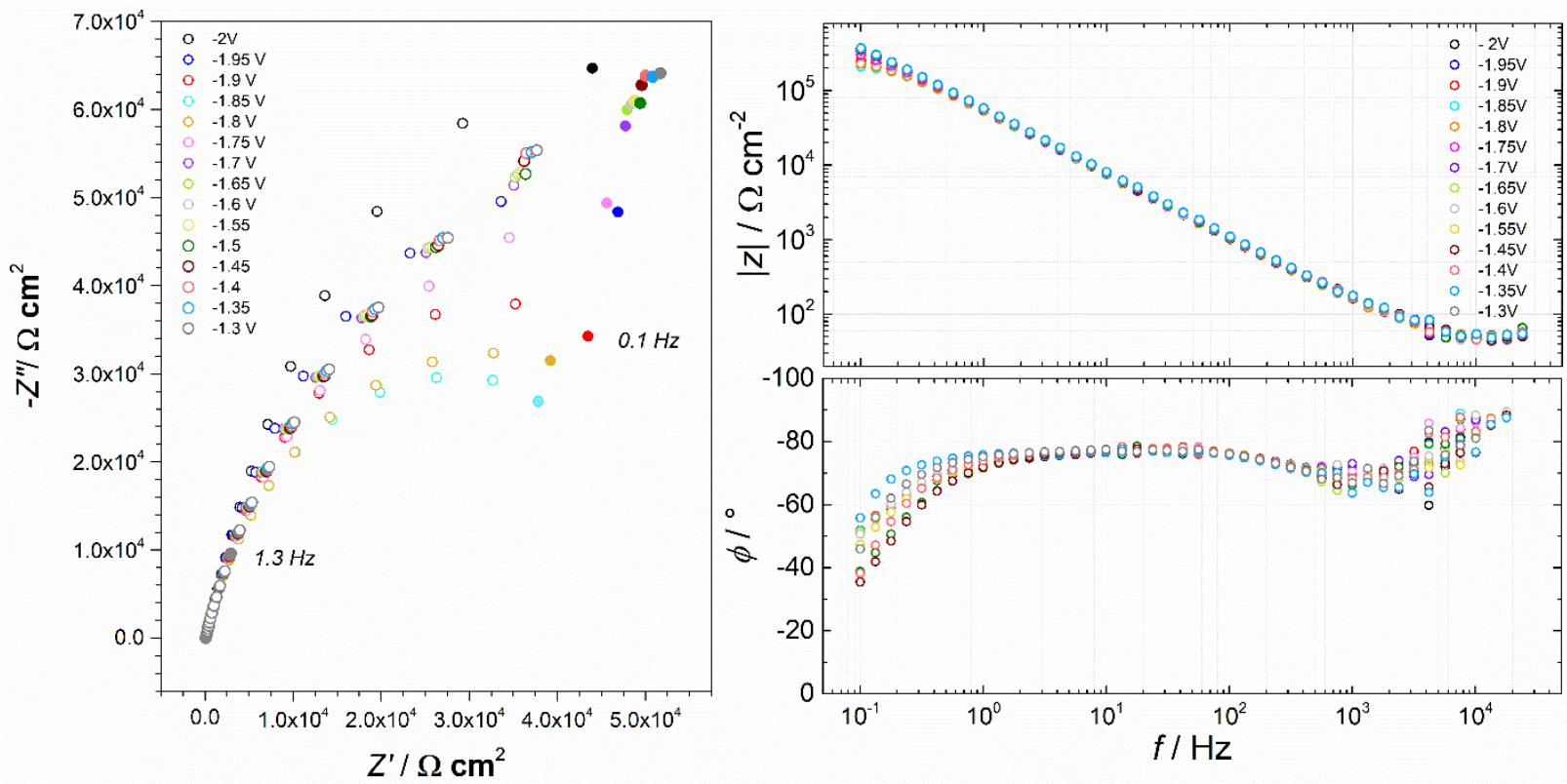

Figure 6: Nyquist and electrolyte resistance corrected Bode representation of the Al electrode in 0.01 $\mathrm{M} \mathrm{Na}_{2} \mathrm{SO}_{4}$ collected at potential varying between -2 to $-1.3 \mathrm{~V}_{\mathrm{MSE}}$ for which the following parameters $\alpha=$ 0.87 and $Q=18 \times 10^{-6} \mathrm{Fs}^{\alpha-1} \mathrm{~cm}^{-2}$ were determined for the CPE describing the double layer capacitance. 


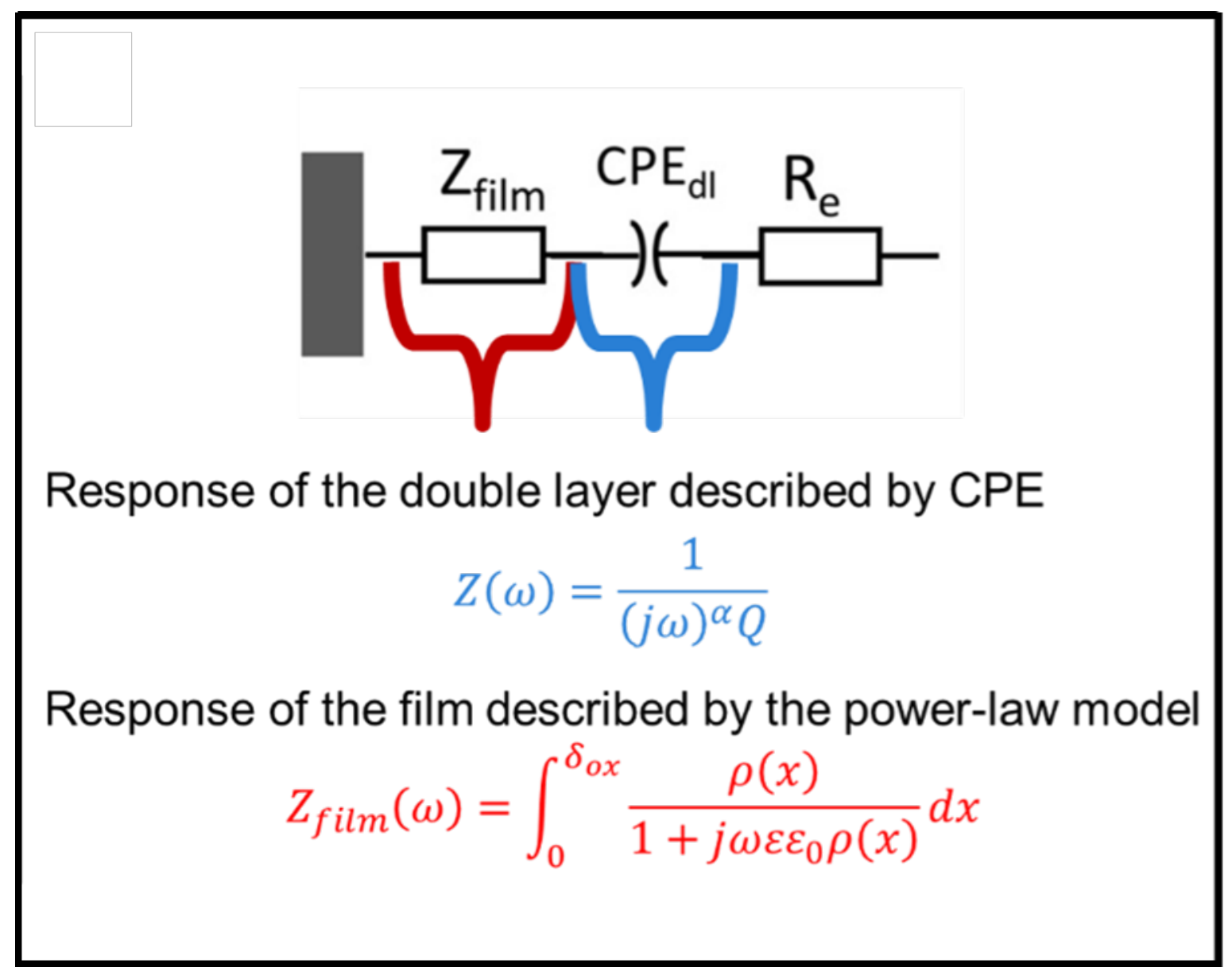

Figure 7: Schematic representing the equivalent circuit of the Al electrode consisting of the impedance of the film $\mathrm{Z}_{\mathrm{film}}$, the double layer capacitance described by a CPE $\mathrm{dl}$ in series with the electrolyte resistance $\mathrm{R}_{\mathrm{e}}$. The double layer and film responses are described by a CPE and a power-law model respectively. 
(a)

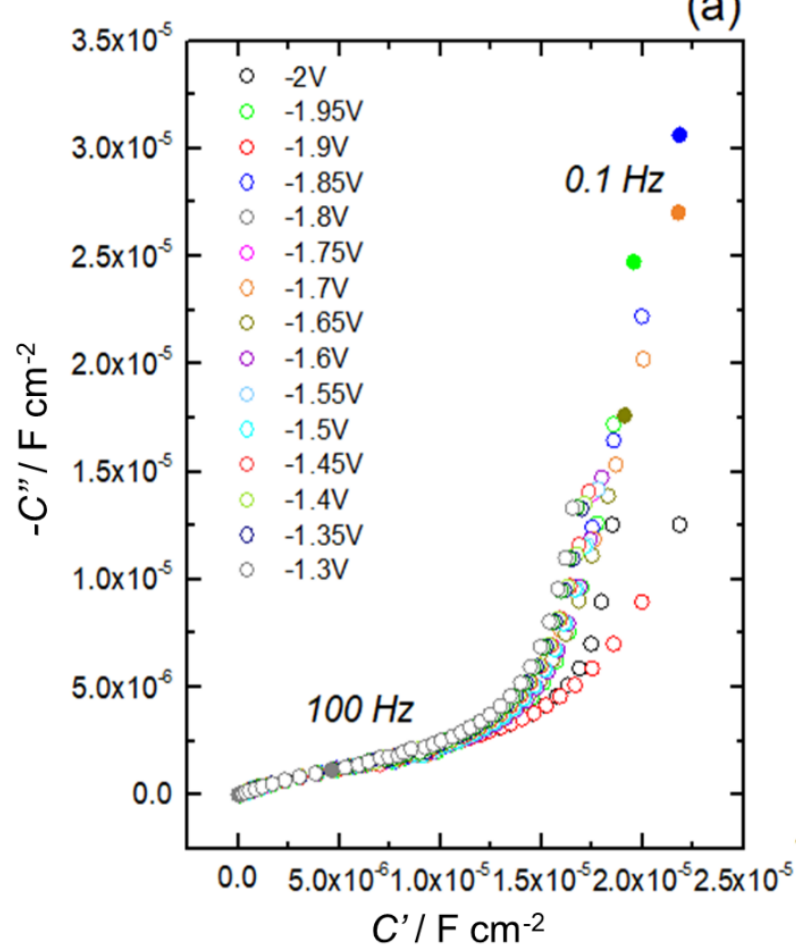

(b)

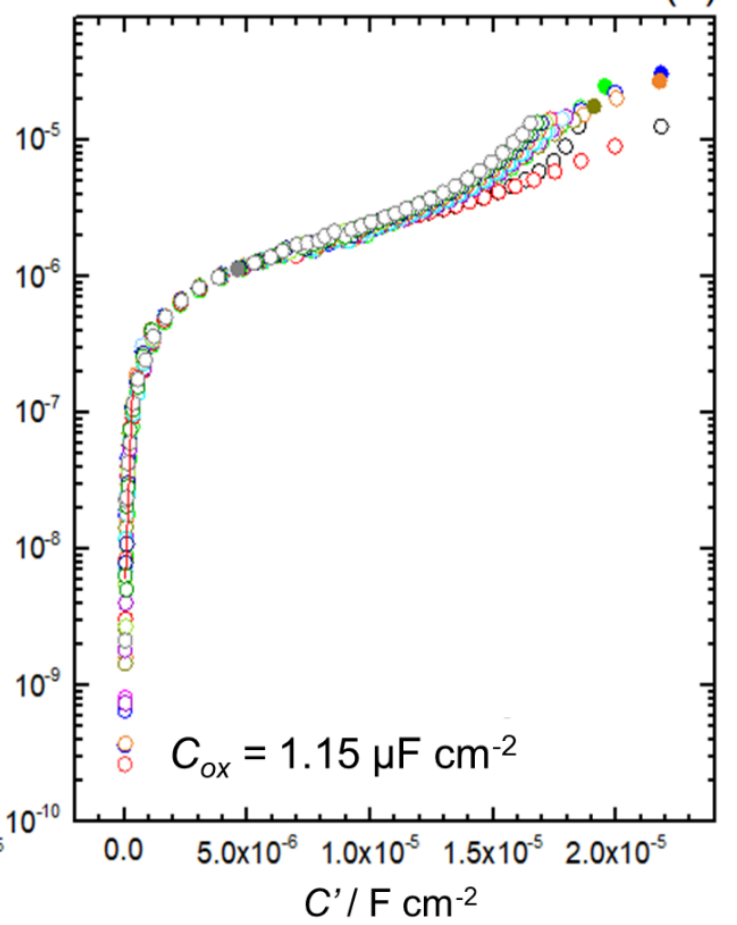

Figure 8: Al in $0.01 \mathrm{M} \mathrm{Na}_{2} \mathrm{SO}_{4}$ - Cole-Cole representation of the complex capacitance (a) and semi logarithmic representation of the complex capacitance determined at potentials varying from $-2 \mathrm{~V}_{\mathrm{MSE}}$ to $-1.3 \mathrm{~V}_{\mathrm{MSE}}$. Herein, the value of the capacitance at infinite frequency is assimilated as the oxide film capacitance $C_{o x}$, determined remains constant with a value of $C_{o x}=1.15 \mu \mathrm{F} \mathrm{cm}$. 


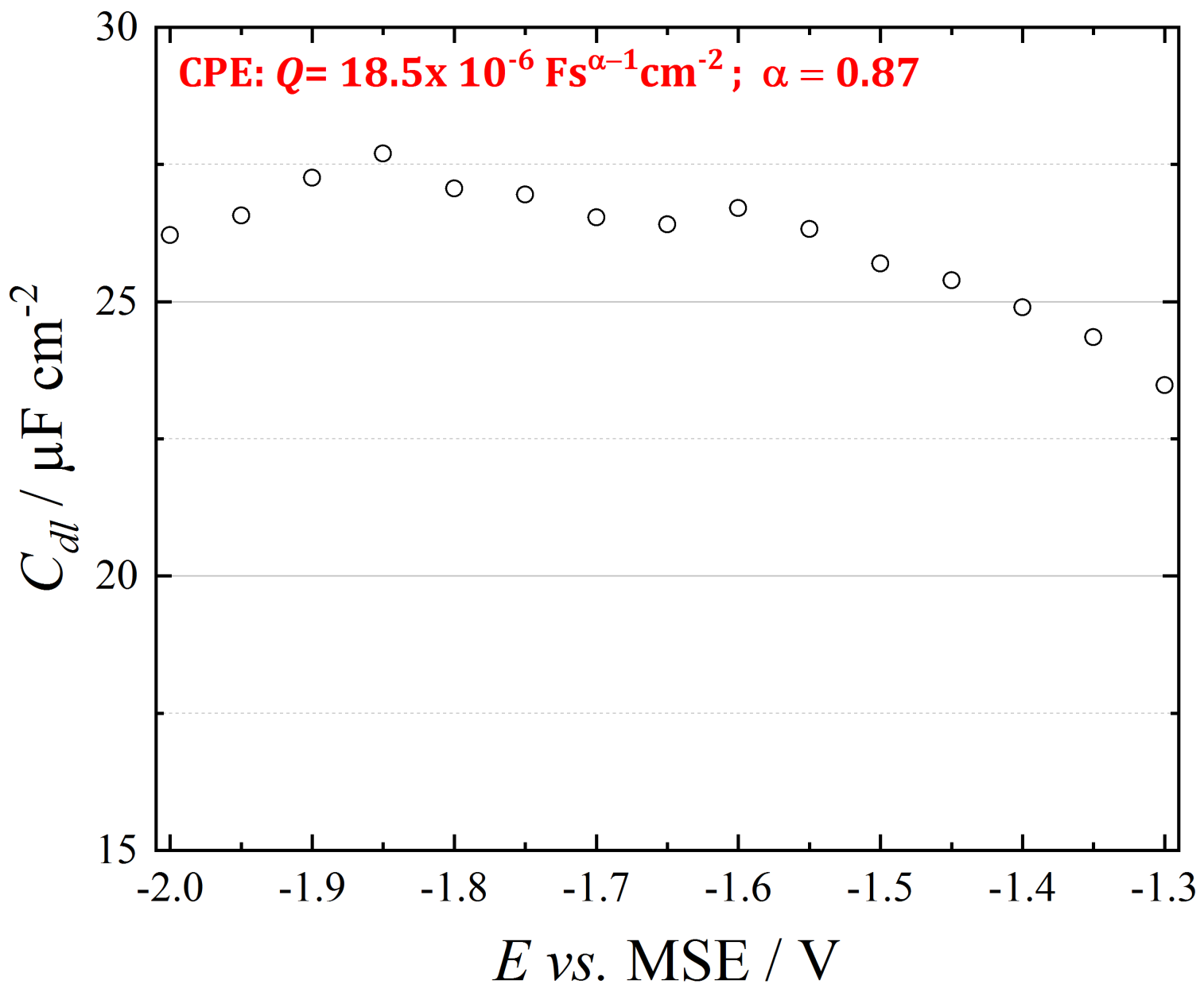

Figure 9: Al in $0.01 \mathrm{M} \mathrm{Na}_{2} \mathrm{SO}_{4}$ - Evolution of the double layer capacitance $C_{d l}$ determined from Brug's equation for each impedance diagram collected between, $-2 \mathrm{~V}_{\mathrm{MSE}}$ and $-1.3 \mathrm{~V}_{\mathrm{MSE}}$. 

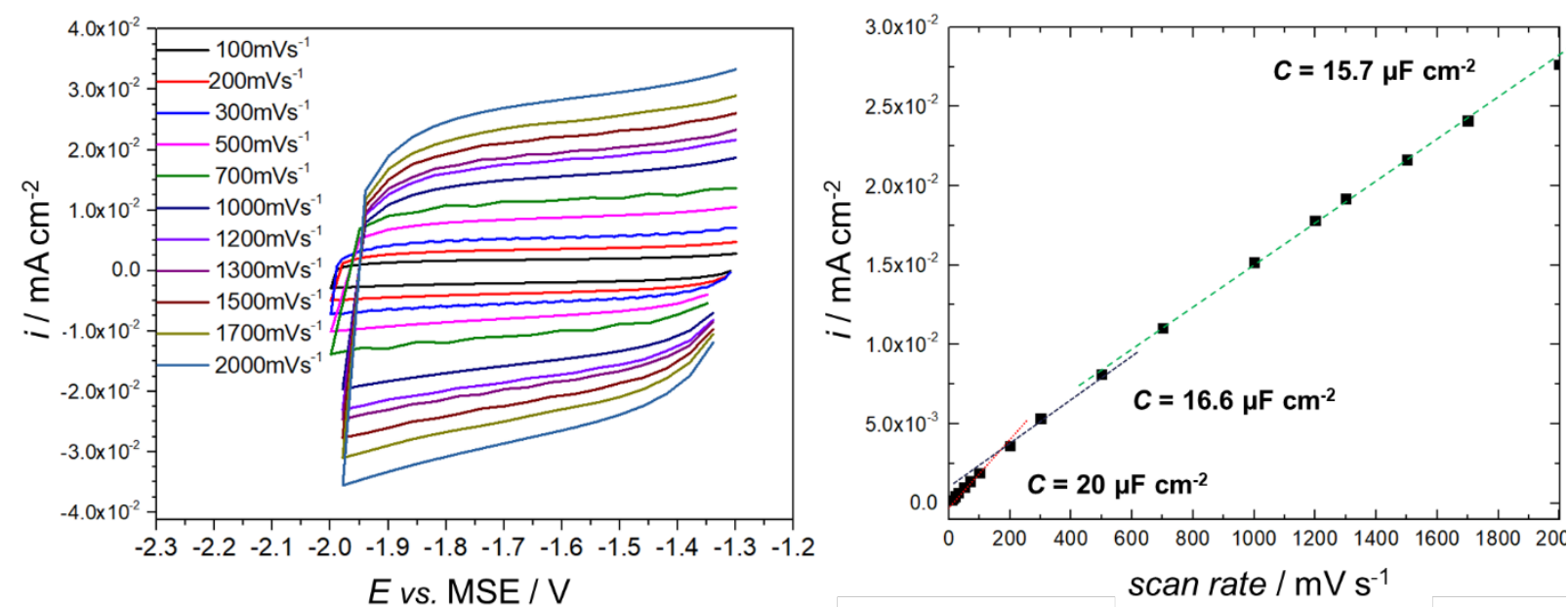

Figure 10: Experimental $\mathrm{CV}$ curves of an $\mathrm{Al}$ electrode in $0.01 \mathrm{M} \mathrm{Na}_{2} \mathrm{SO}_{4}$ recorded at scan rates between 100 and $2000 \mathrm{mV} \mathrm{s}^{-1}$ (scan rates between $1-70 \mathrm{mVs}^{-1}$ not shown here) (a) and the capacitive current $\mathrm{i}_{\mathrm{c}}$, as a function of the scan rate (b). The variation of the linearity between the capacitive current and the scan rate range can be observed as well. 


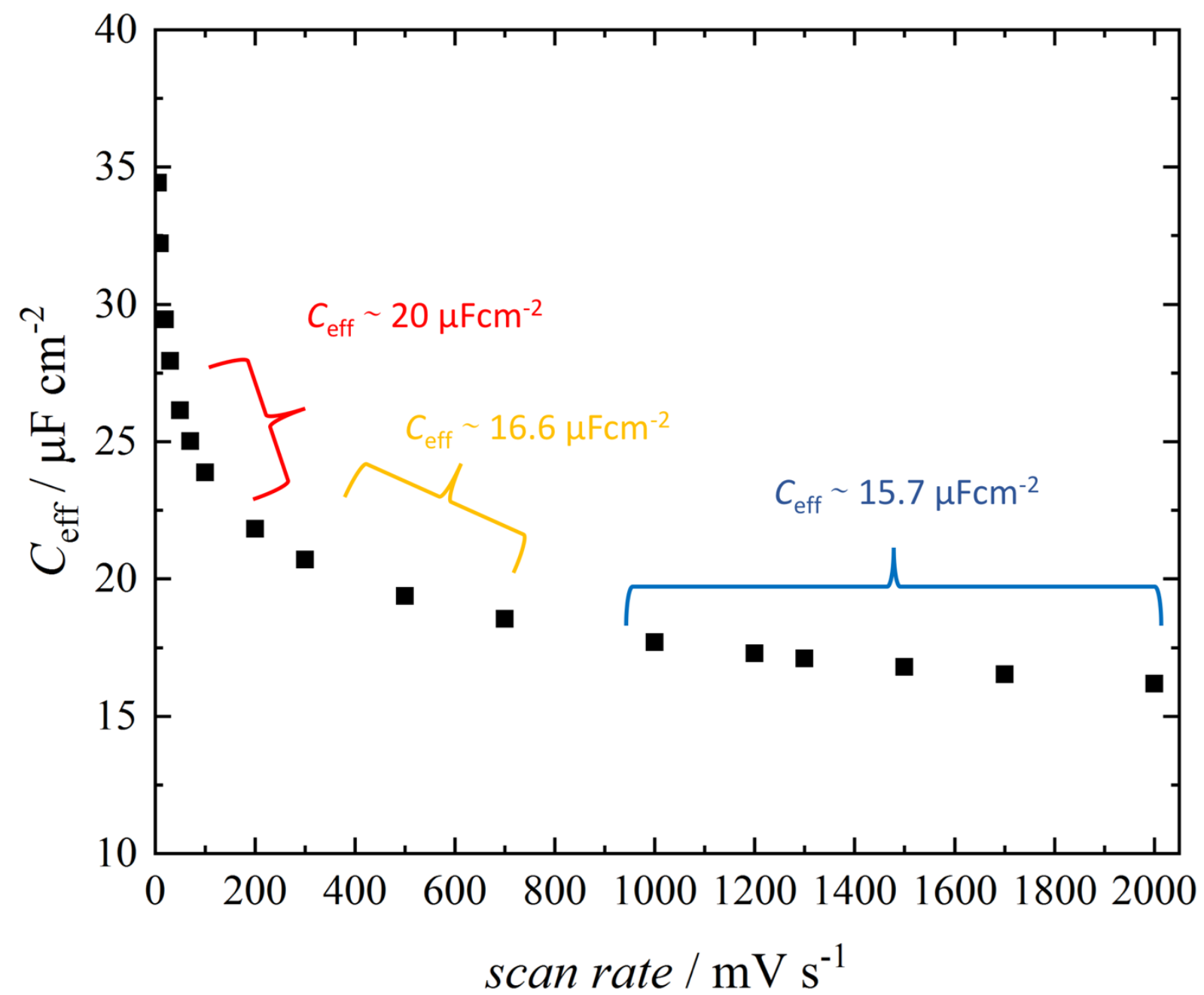

Figure 11: Calculated effective capacitance $C_{e f f}$ as a function of the scan rate. $C_{\text {eff }}$ was determined using the equation from Allagui et al. introducing a CPE element [11] with the $Q$ and $\alpha$ values determined from EIS analysis $\left(\alpha=0.87\right.$ and $\left.\mathrm{Q}=18 \times 10^{-6} \mathrm{Fs}^{\alpha-1} \mathrm{~cm}^{-2}\right)$. The results show good correlation in the values between scan rate ranges varying from $\sim 100 \mathrm{mVs}^{-1}$ and $2 \mathrm{~V} \mathrm{~s}^{-1}, C_{\text {eff }}$ determined from $\mathrm{CV}$ analysis from Figure 10. 